\title{
LA INTELIGENCIA DE NEGOCIOS: HERRAMIENTA CLAVE PARA EL USO DE LA INFORMACIÓN Y LA TOMA DE DECISIONES EMPRESARIALES
}

\section{BUSINESS INTELLIGENCE: A KEY TOOL FOR THE USE OF INFORMATION AND BUSINESS DECISION-MAKING}

Alejandra de-Jesús García-Jiménez ${ }^{1}$ (D); Norma Aguilar-Morales ${ }^{2}$ (D); Leonardo Hernández-Triano ${ }^{3}$ (D); Eduardo Lancaster-Díaz ${ }^{4}$ * (iD).

1. Universidad Juárez Autónoma de Tabasco, Tabasco, México.phd.garcia.alejandra@gmail.com

2. Universidad Juárez Autónoma de Tabasco, Tabasco, México. gialca@hotmail.com

3. Universidad Juárez Autónoma de Tabasco, Tabasco, México. leonardo.hernandez.triano@gmail.com

4. Universidad Juárez Autónoma de Tabasco, Tabasco, México. elancasterna@gmail.com

*Correspondencia del Autor: Alejandra de-Jesús García-Jiménez, correo electrónico: phd.garcia.alejandra@gmail.com.

\section{RESUMEN}

El entorno actual donde las empresas pequeñas y medianas se desarrollan, ha cambiado a raíz del efecto de apertura comercial surgido posterior a la segunda guerra mundial. El objetivo de este documento es analizar la aplicación de la Inteligencia de negocios como una herramienta clave para que las pequeñas y medianas empresas utilicen la información como un insumo de apoyo a la toma de decisiones empresariales. La metodología empleada fue la revisión documental como herramienta, con un enfoque cualitativo descriptivo en el que se utilizaron bases de datos científicas como Dialnet, ScienceDirect y Scielo; y palabras clave como Inteligencias de Negocios, Toma de decisiones, Información. Los criterios de inclusión son aquellos documentos que describan los atributos de la Inteligencia de Negocios, Toma de decisiones e Información para realizar un análisis teórico, crítico y comparativo de las aportaciones de los autores. Los resultados indican que la Inteligencia de Negocios tiene los siguientes componentes: proceso interactivo, explorar, analizar, almacenamiento, tecnología y comunicar. Se concluye que la Inteligencia de Negocios es una solución valorable y viable para las organizaciones pues a través de su uso y aplicación proveen un soporte de conocimiento a los tomadores de decisiones.

Palabras clave: Inteligencia de negocios; información; toma de decisiones.

Cómo citar:

García-Jiménez, Alejandra de-Jesús; Aguilar-Morales, Norma; Hernández-Triano, Leonardo; LancasterDíaz, Eduardo. (2021). LA INTELIGENCIA DE NEGOCIOS: HERRAMIENTA CLAVE PARA EL USO DE LA INFORMACIÓN Y LA TOMA DE DECISIONES EMPRESARIALES. Revista de Investigaciones Universidad del Quindio, 33(1), 132-139. https://doi.org/10.33975/riuq.vol33n1.514

Información del artículo: Recibido: 14 mayo 2021; Aceptado: 18 junio 2021

Revista de Investigaciones Universidad del Quindío, 33(1), 132-139; 2021.

ISSN: 1794-631X e-ISSN: 2500-5782 


\begin{abstract}
In the current environment where small and medium-sized companies develop, it has changed as a result of the commercial opening that took place after the Second World War. The objective of this article is to analyze the importance of applying Business Intelligence as a key tool for small and medium-sized companies to use information as an input to support business decision-making. The methodology used is through a document review with a tool, with a descriptive qualitative approach. Scientific databases such as Dialnet, ScienceDirect and Scielo and keywords such as Business intelligence, Decision making, Information were used to carry out a theoretical, critical and comparative analysis of the contributions of the Business Intelligence authors. The results indicate that Business Intelligence has the components of an interactive process, explore, analyze, data warehouse, technology and communicate. It is concluded that Business Intelligence is a valuable and viable solution for organizations because through its use and application they provide decision makers with the facility to make decisions supported by knowledge.
\end{abstract}

Keywords: Business intelligence; information; decision making.

\section{INTRODUCCIÓN}

El entorno actual en el que las empresas pequeñas y medianas (PYMES) se desarrollan no es el mismo que hace 90 años. Esto, debido a que después de la segunda guerra mundial, se inició un cambio en el sistema económico internacional en el que se liberaron las economías internacionales, se desarrollaron instituciones de cooperación financiera internacional, se incrementó el movimiento de capitales y personas, así como acuerdos de cooperación entre naciones, lo que se conoce como globalización económica (Coppelli, 2018).

Con el surgimiento de la globalización florece también la revolución tecnológica, la cual constituye un impulsor de cambio debido a que los avances en la tecnología han permitido intercambiar información sin importar la geografía, apuntando hacia una nueva sociedad del conocimiento en el que los servicios y las telecomunicaciones son considerados un motor para el crecimiento mundial (Lerner, 1999). Con el desarrollo de la informática y las telecomunicaciones las empresas notaron que las tecnologías y los sistemas de información les permitía lograr la eficiencia en la realización de tareas, ahorrar tiempo para desarrollar sus actividades, almacenar más información en menos espacio, lograr una ventaja competitiva frente a sus competidores, de este modo los sistemas de información se logran establecer como una parte estratégica a considerarse por la planificación empresarial (Hernández, 2003).

La información es considerada un punto clave para la toma de decisiones empresariales, en ella descansa el soporte para los diferentes sistemas de información, recursos financieros, materiales y humanos que integran a la gestión empresarial por lo tanto también debe gestionarse (Muñiz, 2018). Por su parte, el autor Choo (1998) citado por Rodríguez comentó que "en la mayoría de las situaciones, no poseemos una información completa sobre todas las opciones factibles, o no podemos permitirnos el tiempo y el costo de obtener ese conocimiento" (2015, p. 151). Muñiz (2018) plantea que es a través de la aplicación de la inteligencia de negocios como las empresas pueden apoyarse para la toma de decisiones, ya que esta le servirá a la hora de simular diversos escenarios, realizar pronósticos, crear informes, y evaluar posibilidades logrando una clara ventaja competitiva frente a sus competidores.

El objetivo es analizar la importancia de aplicar la Inteligencia de negocios como una herramienta 
clave para que las pequeñas y medianas empresas utilicen la información como un insumo para apoyar la toma de decisiones empresariales. La utilidad del documento es presentar el uso de la inteligencia de negocios en los empresarios PYMES.

\section{Desarrollo del problema}

La información es la clave para tomar decisiones que inciten obtener una ventaja competitiva en el mundo de los negocios, de acuerdo con Muñiz (2018) los tomadores de decisiones necesitan entonces poder acceder a esta lo más rápido, fácil y veraz posible. Entonces esta capacidad de tomar decisiones rápidas debe estar cimentadas en un conocimiento adecuado de la realidad que la empresa posee, aunado a eso conocer su mercado y las tendencias de los mismos (Medina-Chicaiza et al., 2016). Conocer estos datos y convertirlos en conocimiento y el conocimiento en acciones es referirse al proceso de Inteligencia de negocios (Muñiz, 2018). Es por eso que se plantea que las organizaciones deben contar con datos e información para utilizarla como un insumo en la toma de decisiones empresariales apoyada de la herramienta Inteligencia de Negocios.

\section{JUSTIFICACIÓN}

Hoy en día las empresas destinan tiempo y recursos en buscar, procesar, aplicar y proyectar información, puesto que la información es considerada un recurso vital y patrimonio de la organización (Calzada y Abreu, 2009). La información se obtiene a través de un proceso el cual se llama Data Warehouse y es el proceso de extraer datos de aplicaciones internas y externas para ser almacenados en un depósito para su análisis del negocio (Rosado y Rico, 2010). Con el avance de la tecnología y la disminución de los costos de almacenar información, tener grandes cantidades de información a la disposición no resultan tan importante como lo es poder aprovecharla para generar conocimiento (Noriega et al., 2015)
La empresa moderna es entendida como un ente con una organización compleja en el que existen una constante interrelación entre los factores externos e internos los cuales influyen en su actividad y decisiones (Cordero, 2016). Las empresas tienen datos, pero carecen de información (Leyva et al., 2019). Las empresas necesitan establecer elementos que generen valor a través de la adquisición y el manejo del conocimiento (Tello y Velasco, 2016). Es por esto que, la gestión del conocimiento se ha convertido en uno de los principales temas de investigación para promover el valor de los intangibles como centro de las acciones y estrategias empresariales (Rodríguez, 2015). Resulta entonces necesario que las empresas desarrollen la Inteligencia de Negocios a la que Hatch (2009) la define como:

La combinación de prácticas, capacidades y tecnologías usadas por las compañías para recopilar e integrar la información, aplicar reglas del negocio y asegurar la visibilidad de la información en función de una mejor comprensión del mismo $\mathrm{y}$, en última instancia, para mejorar el desempeño (pp. 103-104)

\section{MARCO TEÓRICO}

En la década de los noventa, y conforme las empresas se volvieron más robustas, las organizaciones empezaron a ser parte de la globalización, y la competencia fue cada vez más atroz, los administradores de los negocios se desesperaron por obtener información que lograra la supervivencia de las empresas y mantenerse competitivos. El almacenamiento de datos es una nueva propuesta destinada a brindar información estratégica vital a las organizaciones, fue en esta época que las empresas comenzaron a cimentar ventajas competitivas a través de la construcción de sistemas de almacenamiento de datos (Ponniah, 2004).

Actualmente la gran parte de las empresas cuentan con sistemas de información que soportan una gran parte de las actividades o procesos propios 
del sector de negocios en donde la empresa se desempeña, dichos sistemas pueden ser sencillos o robusto dependiendo los requerimientos que el negocio tengan, con el transcurrir del tiempo estas aplicaciones o sistemas logran acumular la historia de toda la organización, almacenan datos que son utilizados para discutir decisiones que se requieran tomar (Rosado, 2010).

\section{Inteligencia de Negocios}

El investigador de IBM Hans Peter Luhn en el año de 1958 publicó por primera vez en la revista IBM Journal el término Business Intelligence System para aludir a un sistema que sea automático el cual recibe información y la distribuye oportunamente a los lugares adecuados (Rosado et al., 2010). Para el año de 1990 Howard Dresner es considerado como el padre de la Inteligencia de Negocios la concibe como "los conceptos y métodos para mejorar la toma de decisiones empresariales mediante el uso de sistemas basados en hechos de apoyo" (Noriega et al., 2015, p. 304). Desde un punto de vista operativo, Azita (2011) citado de Murillo y Cáceres (2013) describe que la Inteligencia de Negocios conduce a la empresa hacia una ventaja competitiva a través de la información privilegiada y propicia la resolución de los problemas del negocio. De la misma forma, el Data Warehousing Institute TDWI (2015) describe que la Inteligencia de Negocios (BI) es la combinación de un almacén de datos, la tecnología como herramienta de BI, el análisis y la aportación del conocimiento humano para lograr decisiones del negocio óptimas dirigiendo la empresa al éxito (Noriega et al., 2015).

\section{Toma de decisiones}

En cualquier parte de la vida humana y las actividades que se desarrollan, la toma de decisiones es fundamental. Pero cabe mencionar que tomar decisiones acertadamente requiere un proceso de razonamiento arduo y enfocado, además de poder converger el uso de varias disciplinas. Por el lado empresarial, un gerente o administrador toma muchas decisiones diariamente, algunas son rutinarias, pero otras son de carácter crítico en las cuales se ve involucrado la generación de ganancia o la pérdida de dinero o incumplir con compromisos que la organización ya tenía pactados previamente. Con frecuencia las decisiones rutinarias son tomadas inconscientemente y con rapidez, mientras que las complejas se requiere tomar un tiempo de análisis y realizar un proceso sistemático (Amaya, 2010).

El proceso de toma de decisiones considera las cuatro funciones administrativas, y a los administradores que planean, organizan, dirigen y controlan se les llama tomadores de decisiones. El tomar una decisión implica elegir una alternativa de todas las posibles existentes. Para llevar a cabo este proceso se requiere de información, porque cada alternativa considera una consecuencia con respecto al objetivo que se quiere lograr, y es aquí la importancia de la información que es el proceso de transformar la información en acción (Castro, 2013). El concepto de acción de Alfred Schutz toma en consideración que la acción comienza con un proceso mental y termina en la realización de un acto correspondiente, lo que significa la intervención del objetivo, el plan mental, las alternativas y el acto (Vélez, 2006).

\section{METODOLOGÍA}

De acuerdo con Denzin y Lincoln (2011) citado de Hernán-García et al. (2020) "en investigación cualitativa, la interpretación personal para la recopilación de datos es muy relevante" (p. 2). Este documento se realiza bajo un enfoque cualitativo descriptivo en el que se utiliza como técnica la revisión documental. Existen herramientas como el internet y fuentes de información como las bases de datos para poder llevar a cabo investigaciones en el tiempo donde se reduce la movilidad (Hernán-García et al., 2020). En la investigación realizada para este artículo utilizamos bases datos científicas las cuales son: Dialnet, ScienceDirect y Scielo. Las palabras clave para identificar aquellos factores determinantes de una toma de decisiones basada en análisis de información 
y el uso de la inteligencia de negocios como herramienta fueron: Inteligencia de Negocios, Toma de decisiones, Información. El tipo de análisis es teórico, crítico y comparativo de las aportaciones de los autores, en las que se muestre sus convergencias y divergencias para identificar los factores que intervienen en el uso eficiente de la información empresarial y la toma de decisiones, sirviendo de guía para los emprendedores y empresarios de las pequeñas y medianas empresas. A continuación, se presenta la información recolectada previamente seleccionada y analizada que dieron los siguientes resultados.

\section{RESULTADOS}

Los resultados obtenidos de la revisión documental de los artículos científicos que cumplieron con los criterios de búsqueda, se muestran en la siguiente tabla que expresan el autor y su aportación sobre el concepto de Inteligencia de Negocios.

Tabla 1. Aportación de Autores a la conceptualización de la Inteligencia de Negocios

\begin{tabular}{|c|c|c|}
\hline No. & Autor(es) & Aportación(es) sobre Inteligencia de Negocios \\
\hline A1 & Luhn (1958) & $\begin{array}{l}\text { - Habilidad para aprehender las interrelaciones de hechos en tal forma que } \\
\text { guie la acción hacia el objetivo deseado. }\end{array}$ \\
\hline A2 & Dresner (1989) & $\begin{array}{l}\text { - Utilizar sistemas que se basan en hechos de apoyo para mejorar la toma } \\
\text { de decisiones empresariales. }\end{array}$ \\
\hline A3 & Watson (2009) & $\begin{array}{l}\text { - La inteligencia de negocios es una categoría amplia de aplicaciones, } \\
\text { tecnologías y procesos para la recolección, almacenamiento, el acceso y } \\
\text { análisis de datos para ayudar a los usuarios a tomar mejores decisiones } \\
\text { de negocio. }\end{array}$ \\
\hline A4 & Rosa & - Es definida como la habilidad corporativa para tomar decisiones \\
\hline A5 & Azita (2011) & $\begin{array}{l}\text { - La inteligencia empresarial (BI) se refiere a las técnicas informáticas } \\
\text { que se utilizan para detectar, excavar y analizar datos comerciales, como } \\
\text { ingresos por ventas por productos y / o departamentos, o por costos e } \\
\text { ingresos asociados. }\end{array}$ \\
\hline A6 & $\begin{array}{l}\text { Ahumada-Tello } \\
\text { (2012) }\end{array}$ & $\begin{array}{l}\text { - Conjunto de estrategias, acciones y herramientas enfocadas a la } \\
\text { administración y creación de conocimiento mediante el análisis de datos } \\
\text { existentes en una organización o empresa }\end{array}$ \\
\hline A7 & TDWI (2017) & $\begin{array}{l}\text { - La combinación de tecnología, herramientas y procesos que permiten } \\
\text { transformar los datos almacenados en información, esta información en } \\
\text { conocimiento y este conocimiento dirigido a un plan estratégico o una } \\
\text { estrategia comercial. }\end{array}$ \\
\hline
\end{tabular}

- Es un proceso interactivo para explorary analizar información estructurada sobre un área (normalmente almacenada en un data warehouse), para

A8 Gartner Group (2020) descubrir tendencias o patrones, a partir de los cuales derivar ideas y extraer conclusiones. El proceso de BI incluye la comunicación de los descubrimientos y efectuar los cambios. Las áreas incluyen clientes, proveedores, productos, servicios y competidores 


\section{¿Cuál es el valor del conocimiento para las organizaciones?}

Para comprender el concepto se considera importante analizar detenidamente las partes que lo comprenden:

Tabla 2. Componentes de la Inteligencia de Negocios

\begin{tabular}{|c|c|c|c|}
\hline Componente & Acción & Aplicación & \\
\hline Proceso interactivo & $\begin{array}{l}\text { La inteligencia de negocios supone un } \\
\text { análisis de información continua y no } \\
\text { solo un extracto en un momento exacto. }\end{array}$ & $\begin{array}{l}\text { Permitir reunir, depurar } \\
\text { transformar los datos } \\
\text { conocimiento. }\end{array}$ & $\begin{array}{r}\mathrm{y} \\
\text { en }\end{array}$ \\
\hline Explorar & $\begin{array}{l}\text { La inteligencia de negocios facilita } \\
\text { comprender que ocurre en el negocio } \\
\text { y permite acceder a información que } \\
\text { permitirá la interpretación, incluso } \\
\text { descubrir nuevas relaciones. }\end{array}$ & $\begin{array}{l}\text { Detectar las } \\
\text { oportunidades. }\end{array}$ & $\mathrm{y}$ \\
\hline
\end{tabular}

La inteligencia de negocios procura descubrir las variables, sus interacciones

Analizar y las probabilidades de que ocurra nuevamente las relaciones o evolución de las mismas.
Aportar conocimiento relevante para la toma de decisiones correcta en un escenario de incertidumbre.

La inteligencia de negocios se basa en el Almacenar y utilizar herramientas

Data warehouse uso de los datos que están almacenados en los sistemas. de análisis de datos y gestión de contenidos.

La inteligencia de negocios canaliza Soportar las decisiones que se Tecnología hacia un objetivo en concreto. toman a nivel interno y externo.

La inteligencia de negocios comunica lo que se haya descubierto y realiza los Elaborar un plan estratégico Comunicar cambios necesarios en la organización conforme a las necesidades de para mejorar la competitividad y cada organización. obtener la ventaja competitiva.

Nota: Elaboración propia con base en Gómez (2013), Pinto-López y Malcón-Cervera (2018) y Rosado y Rico (2010).

La inteligencia de negocios se conceptualiza por medio de los sistemas de información, mecanismos de innovación y los procesos de toma de decisiones (Tello y Velasco, 2016); así mismo los encargados de tomar decisiones en la organización, requieren contar con acceso a la información precisa y pertinente para lograr alcanzar sus objetivos, para esto la Inteligencia de Negocios integra y cruza los datos para evitar análisis separados o incompletos, y que sus análisis sirva como soporte para la toma de decisiones (Vanegas y Guerra, 2013) y por último la Inteligencia de Negocios brinda trazabilidad en la información la cual permite visualizar las operaciones comerciales históricas, actuales y futuras (Azita, 2011).

\section{CONCLUSIONES}

Ya se ha establecido que el mundo de los negocios cambia rápidamente, $\mathrm{y}$ que las empresas pequeñas y medianas tienen que buscar adaptarse a estos cambios a través del uso y aplicación de herramientas como la Inteligencia 
de Negocios. De acuerdo con Tello y Perusquia es a través de la aplicación de la inteligencia de negocios que se puede establecer como una principal ventaja competitiva en la empresa. La información entonces se vuelve una herramienta para alcanzar los objetivos estratégicos de la organización, secundando una forma de aplicar un proceso de toma de decisiones inteligente. Hoy en día las empresas deben apostar por la implementación de un modelo de Inteligencia de negocios para apoyarse en la toma de decisiones empresariales, la Inteligencia de Negocios se ha convertido en una solución valorable y viable para las organizaciones, pues a través de su uso y aplicación proveen a los tomadores de decisiones la facilidad de tomar decisiones soportadas por conocimiento.

\section{REFLEXIONES Y RECOMENDACIONES}

Las organizaciones están copiosas de datos que fluyen a través de sus canales de comunicación, pero es necesario que los datos se transformen en información y esa información debe a su vez ser transformada en conocimiento, de esta manera se pueden tomar decisiones empresariales basadas en conocimiento. Finalmente, se recomienda que las organizaciones se esmeren a la hora de elegir los sistemas adecuados que sirvan de soporte para la toma de decisiones empresariales, por lo cual el uso y aplicación de la Inteligencia de Negocios logra ser una herramienta adecuada.

\section{REFERENCIAS}

1. Ahumada, E., \& Perusquia, J. (2015). Inteligencia de negocios: estrategia para el desarrollo de competitividad en empresas de base tecnológica. Contaduría y Administración(61), 127-158. doi:http://dx.doi.org/10.1016/j.cya.2015.09.006

2. Azita, S. (2011). An Approach to Building and Implementation of Business Intelligence System in Exchange Stock Companies. Australian Journal of Basic and Applied Sciences, 5(6), 1491-1495.

3. Calzada, L., \& Abreu, J. (2009). El impacto de las herramientas de inteligencia de negocios en la toma de decisiones de los ejecutivos. Daena: International journal of good conscience, 16-52.

4. Coppelli, G. (2018). La globalización económica del siglo XXI. Entre la mundialización y la desglobalización. Estudios Internacionales(191), 57-80.

5. Gómez, A. (2004). Desigualdad salarial: ¿Causa o consecuencia de la integración. Universidad EAFIT, 40(133), 8-21.

6. Gómez, A. (2013). Inteligencia de negocios, una ventaja competitiva para las organizaciones. Revista "Ciencia y Tecnología”, Escuela de Postgrado - UNT, 85-96.

7. Hernández, A. (2003). Los sistemas de información: evolución y desarrollo. Proyecto social Revista de relaciones laborales(10-11), 149-165.

8. Hernán-García, M., Lineros-González, C., \& Ruiz-Azarola, A. (2020). Cómo adaptar una investigación cualitativa a contextos de confinamiento. Gaceta Sanitaria, 1-4. doi:https://doi. org/10.1016/j.gaceta.2020.06.007

9. Lerner, B. (1999). Un panorama general de la globalización: génesis, evolución y perspectivas. Revista de investigaciones politicas y sociológicas, 1(1), 11-40. Obtenido de http://www.redalyc. org/articulo.oa? id $=38010102$

10. Leyva, L., \& Bernabé, D. (2019). Solución de Inteligencia de Negocios que permita una mejor integración de datos y soporte en la toma de decisiones en el proceso de ventas de la empresa librería Crisol S.A.C. Sucursal Trujillo. Tesis.

11. Medina-Chicaiza, R., Chiliquinga-Vejar, L., \& Ortiz-Barba, A. (2016). Aproximación sobre la inteligencia de negocios en las PYME. REVISTA CIENTIFICA DOMINIO DE LAS CIENCIAS, 2(4), 370-382.

12. Muñiz, L. (2018). El Business Intelligence herramienta clave para mejorar la gestión empresarial.

13. Muñoz-Hernández, H., Osorio-Mass, R., \& Zúñiga-Pérez, L. (julio-diciembre de 2016). Inteligencia de los negocios Clave del éxito en la era de la información. Revista Clío América, 10(20). doi:http://dx.doi.org/10.21676/23897848.1877 
14. Murillo, M., \& Cáceres, G. (2013). Business intelligence y la toma de decisiones financieras: una aproximación teórica. Revista Logos, Ciencia \& Tecnología, 5(1), 119-138. doi:https://doi. org/10.22335/rlct.v5i1.11

15. Noriega, R., Valdivia, M., Valenzuela, J., Tamer, M., \& Acosta, J. (2015). Evolución de la inteligencia de negocios. Culcyt, 12(57), 299-308.

16. Rodríguez, Y. (2015). Gestión de Información y del Conocimiento para la toma de decisiones organizacionales. bibliotecas anales de investigación, 11, 150-163.

17. Rosado, A., \& Rico, D. (Abril de 2010). Inteligencia de Negocios: Estado del Arte. Scientia et Technica, XVI(44), 321-326.

18. Vanegas, E., \& Guerra, L. (2013). Sistema de inteligencia de negocios para el apoyo al proceso de toma de decisiones. Revista INGENIERÍA UC, 20(3), 25-34. 